\title{
A Detailed Investigation of the Onion Structure of Exchanged Coupled Magnetic $\mathrm{Fe}_{3-\delta} \mathrm{O}_{4} @ \mathrm{CoFe}_{2} \mathrm{O}_{4} @ \mathrm{Fe}_{3-\delta} \mathrm{O}_{4} \mathrm{Nanoparticles}$
}

Kevin Sartori, ${ }^{1,2,3}$ Anamaria Musat, ${ }^{1}$ Fadi Choueikani, ${ }^{2}$ Jean Marc Grenèche, ${ }^{4}$ Simon Hettler, ${ }^{5,6}$ Peter Bencok, ${ }^{7}$ Sylvie Begin-Colin, ${ }^{1}$ Paul Steadman, ${ }^{7}$ Raul Arenal, ${ }^{5,6,8}$ Benoit P. Pichon ${ }^{*}, 1,9$

\footnotetext{
${ }^{1}$ Université de Strasbourg, CNRS, Institut de Physique et Chimie des Matériaux de Strasbourg, UMR 7504, F67000 Strasbourg, France

2 Synchrotron SOLEIL, L'Orme des Merisiers, Saint Aubin - BP48, 91192 Gif-sur-Yvette, France

${ }^{3}$ Laboratoire Léon Brillouin, UMR12 CEA-CNRS, F-91191 Gif-sur-Yvette, France

${ }^{4}$ Institut des Molécules et Matériaux du Mans, IMMM, UMR CNRS-6283 Université du Maine, avenue Olivier Messiaen, 72085 Le Mans Cedex 9, France

${ }^{5}$ Instituto de Nanociencia y Materiales de Aragon (INMA), CSIC-Universidad de Zaragoza, Calle Pedro Cerbuna, 50009 Zaragoza, Spain

${ }^{6}$ Laboratorio de Microscopias Avanzadas (LMA), Universidad de Zaragoza, Calle Mariano Esquillor, 50018

Zaragoza, Spain

7 Diamond Light Source, Didcot OX11 0DE, UK

${ }^{8}$ Fundacion ARAID, 50018 Zaragoza, Spain

${ }^{9}$ Institut Universitaire de France, 1 rue Descartes, 75231 Paris Cedex 05, France
}

Corresponding Author

*E-mail: Benoit.Pichon@unistra.fr 


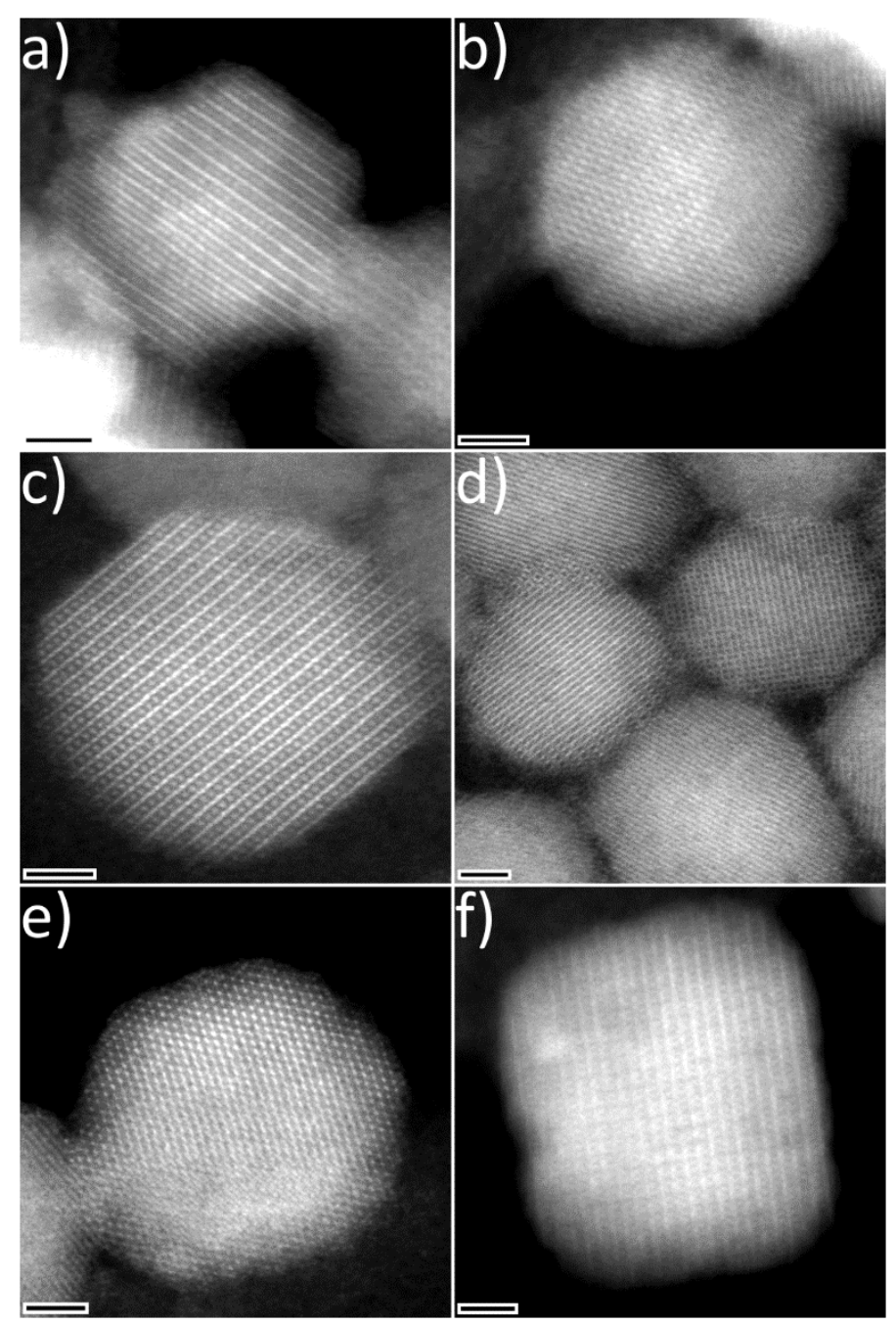

Figure S1. Additional STEM-HAADF micrographs of (a), b) C, (c), d)) CS and (e), f)) CSS nanoparticles. A minor crystal defect in the $C$ nanoparticle in b) may be recognized on the right side. (a) ,b) and f)) are acquired at an electron energy of $80 \mathrm{keV}$. Especially for $\mathrm{C}$ nanoparticles significant damage was observed at an electron energy of $300 \mathrm{keV}$. Scale bars are $2 \mathrm{~nm}$.

a)

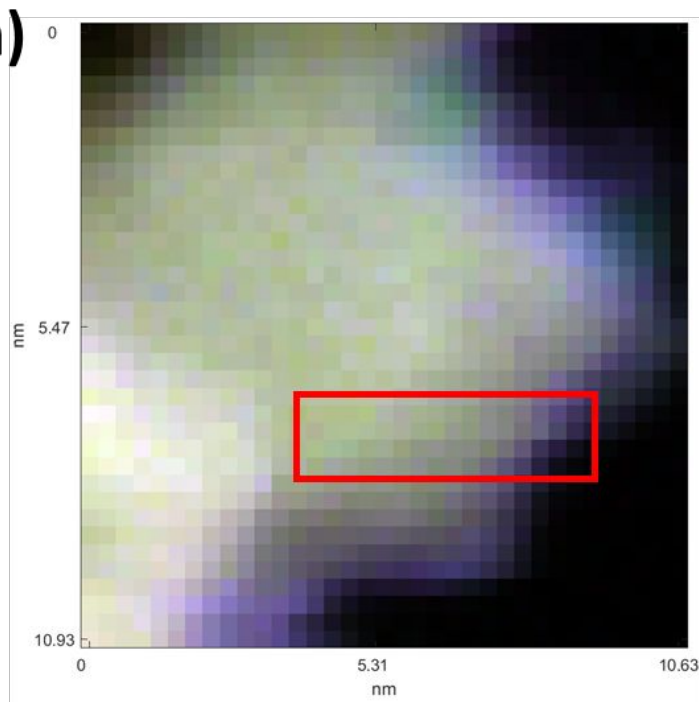

b)

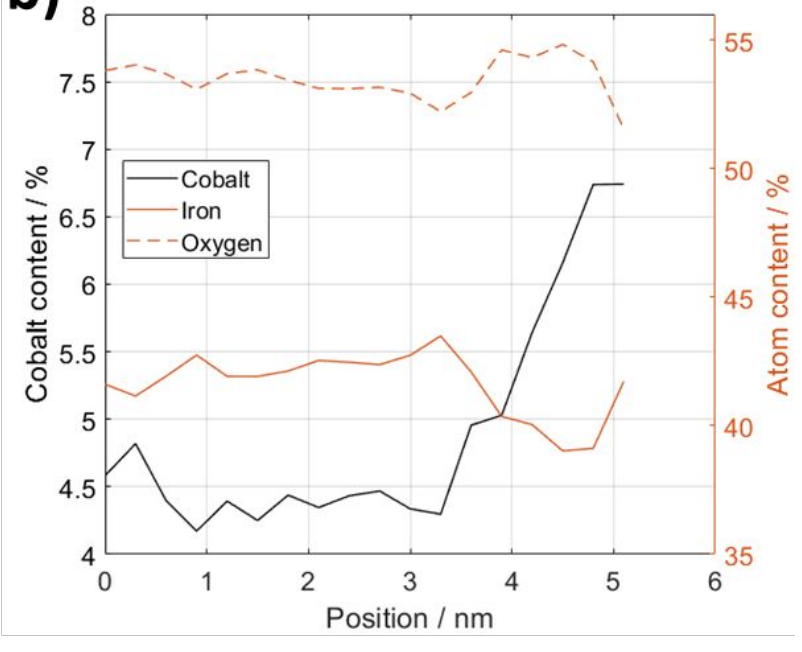

Figure S2. a) EELS-SI mapping of CS nanoparticle, the red rectangle shows the working area to perform the b) plot of the atomic composition. 

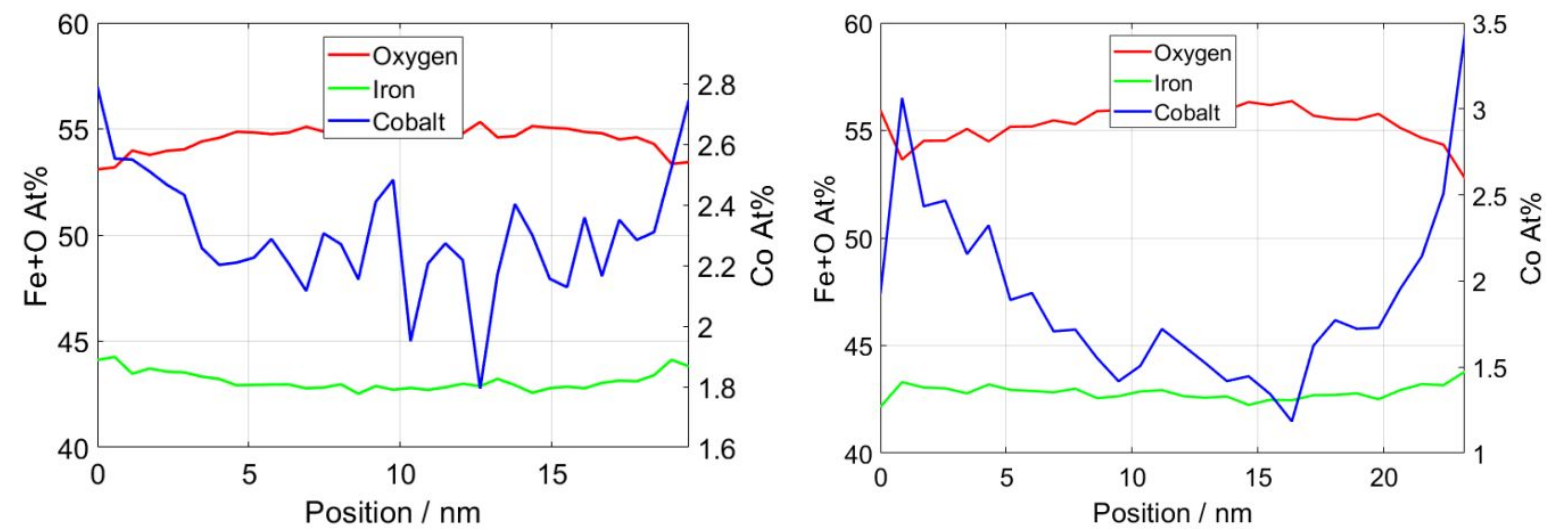

Figure S3. Composition line scans across two CSS nanoparticles showing the slight increase of Co at the nanoparticle edge. The composition at the very edge has to be taken with care due to the very low signal to noise ratio.
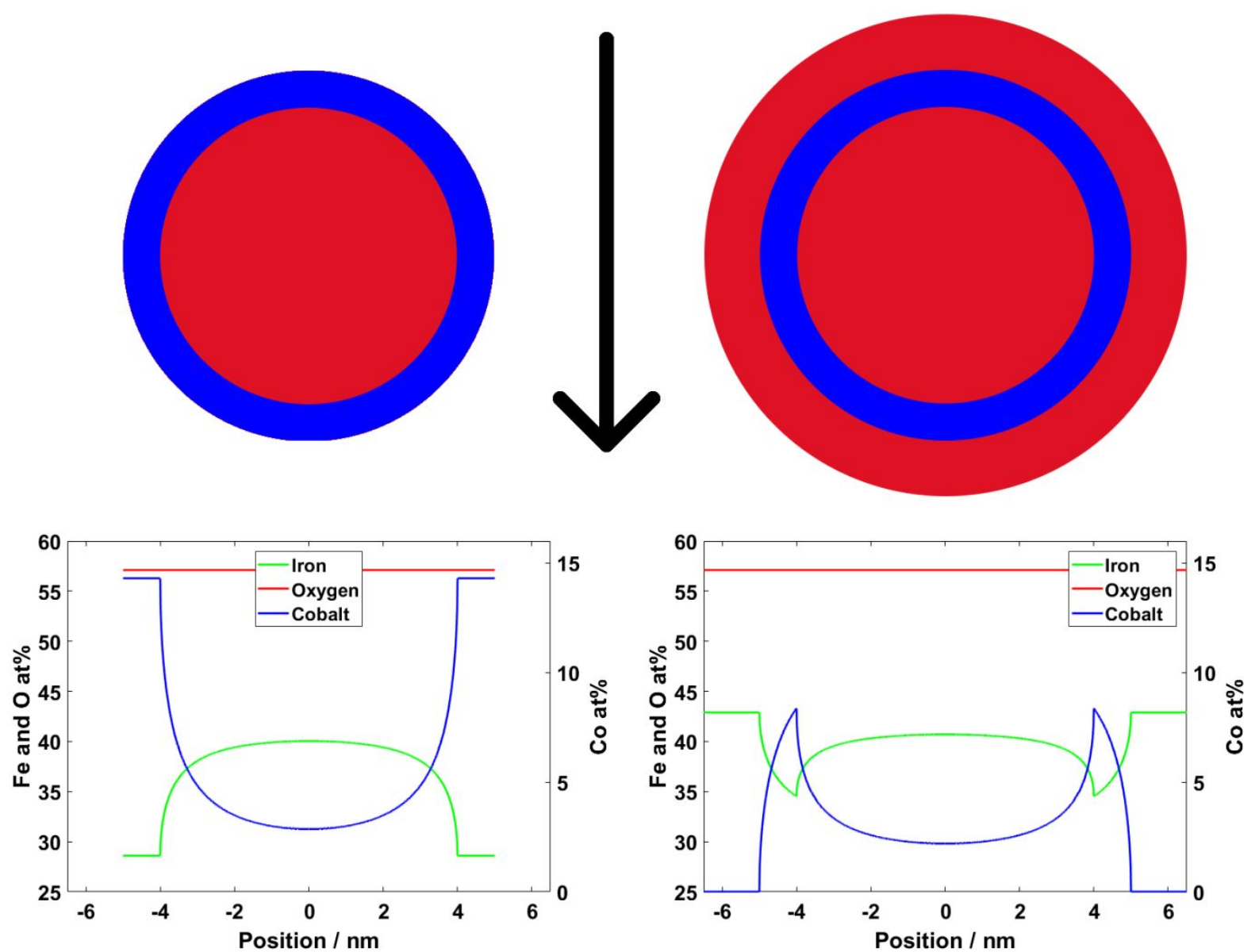

Figure S4. Expected composition profile for a CS (left) and CSS (right) nanoparticle assuming a $\mathrm{Fe}_{3} \mathrm{O}_{4}$ core and $2^{\text {nd }}$ shell (red) and a $\mathrm{CoFe}_{2} \mathrm{O}_{4}$ shell (blue) due to the projection effect in STEM-EELS. 

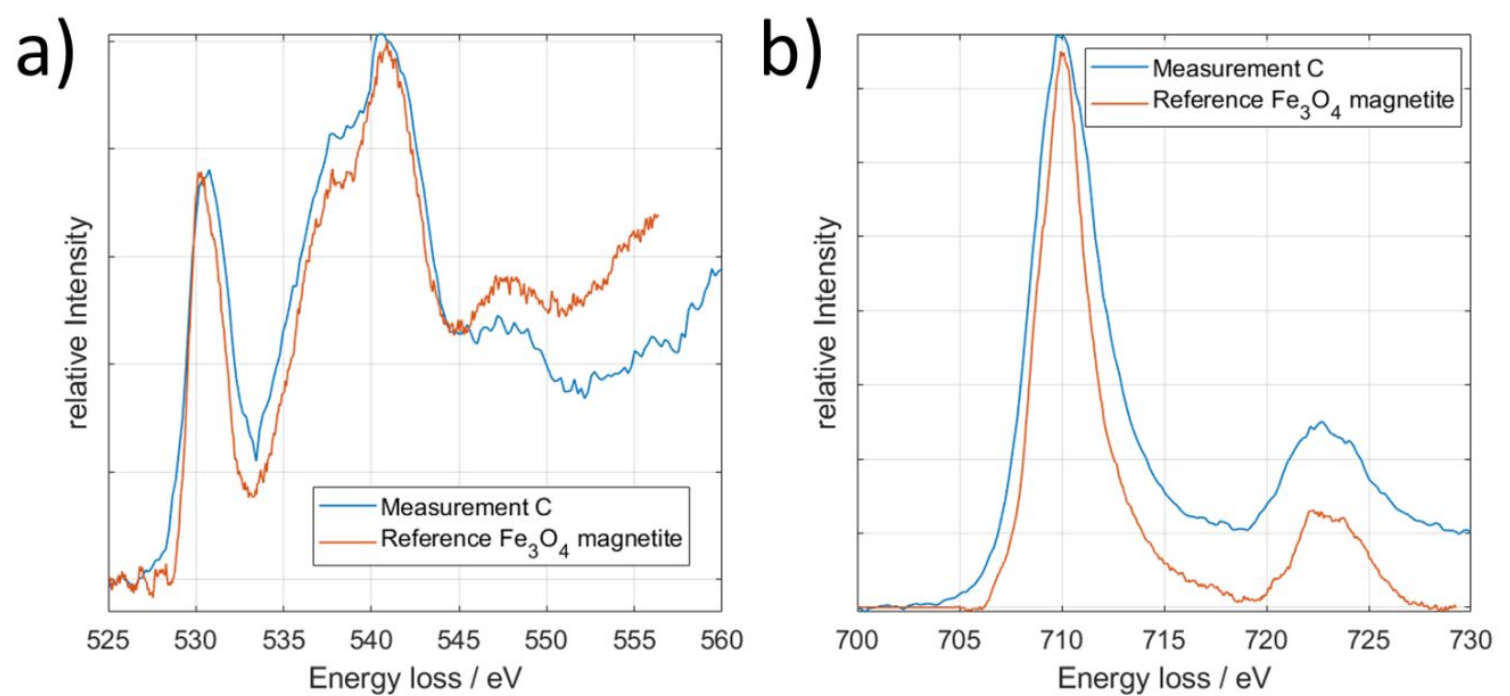

Figure S5. Comparison of exemplary background-subtracted EELS spectra of the a) O-K edge and $\mathrm{b}$ ) the Fe-L edge obtained from the core nanoparticles with a reference for $\mathrm{Fe}_{3} \mathrm{O}_{4}$ magnetite (see reference 48 in the manuscript).
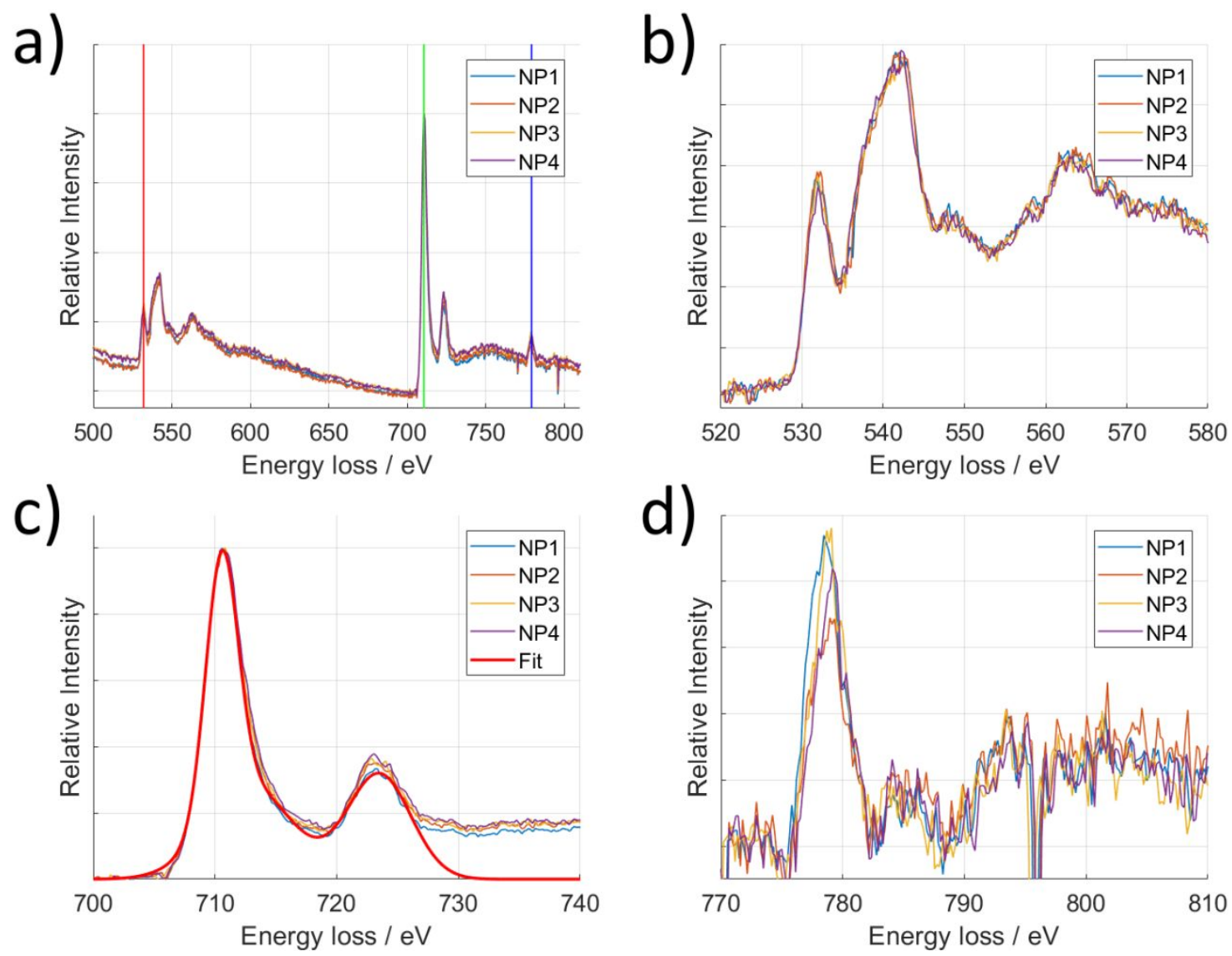

Figure S6. Comparison of EELS spectra of the core region of 4 different CS nanoparticles revealing the high similarity between different nanoparticles in the batch. a) Overview spectrum with O-K (red), Fe-L (green) and Co-L (blue) edges marked. Background-subtracted and normalized (with respect to Fe- $\mathrm{L}_{3}$ peak at $710.5 \mathrm{eV}$ ) spectra of b) O-K edge, c) Fe-L edge with fit based on three Gaussians and d) Co-L edge. 

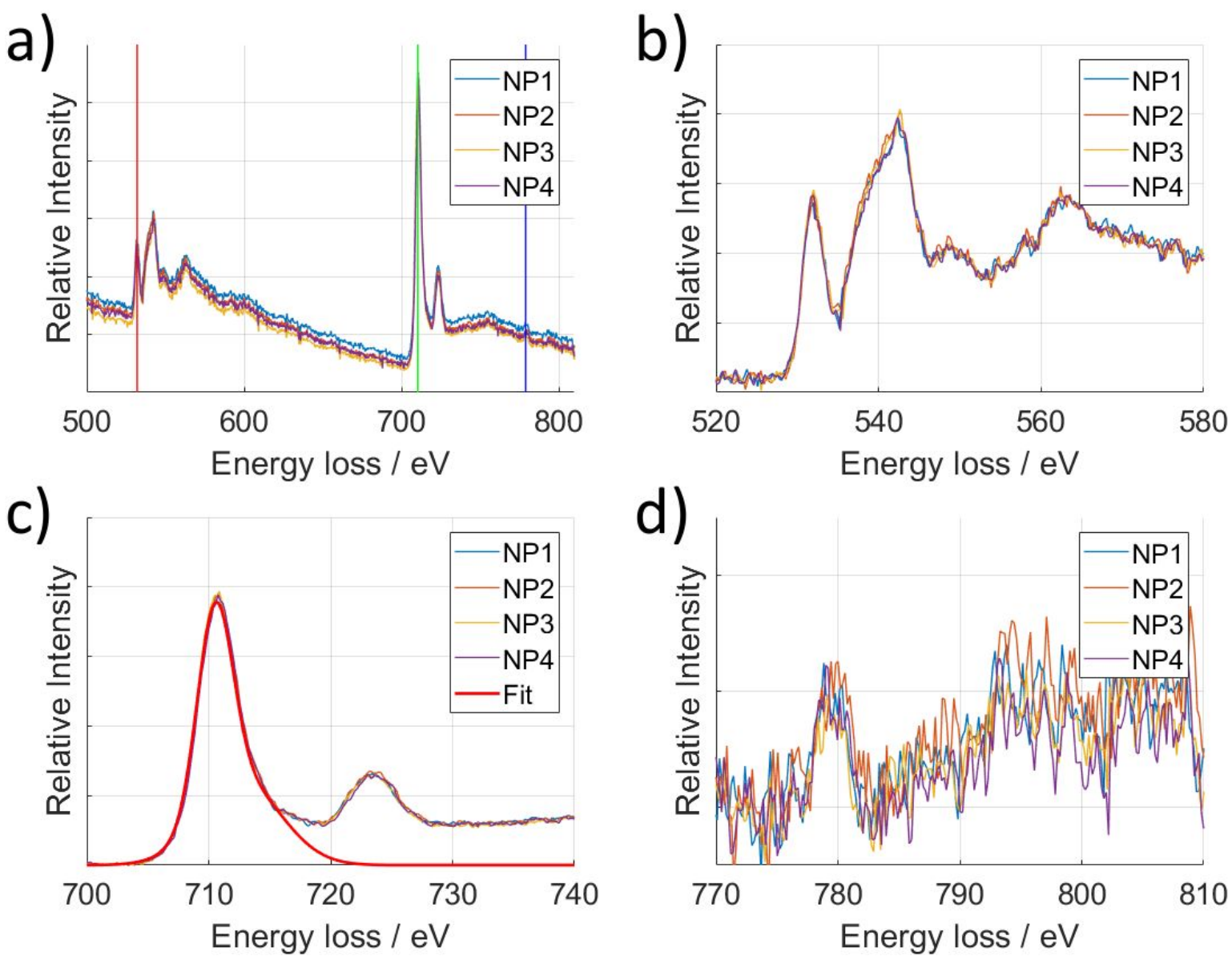

Figure S7. Comparison of EELS spectra of the core region of 4 different CSS nanoparticles revealing the high similarity between different nanoparticles in the batch. a) Overview spectrum with O-K (red), Fe-L (green) and Co-L (blue) edges marked. Background-subtracted and normalized (with respect to Fe- $\mathrm{L}_{3}$ peak at $710.5 \mathrm{eV}$ ) spectra of b) O-K edge, c) Fe-L edge with fit based on two Gaussians and d) Co-L edge.
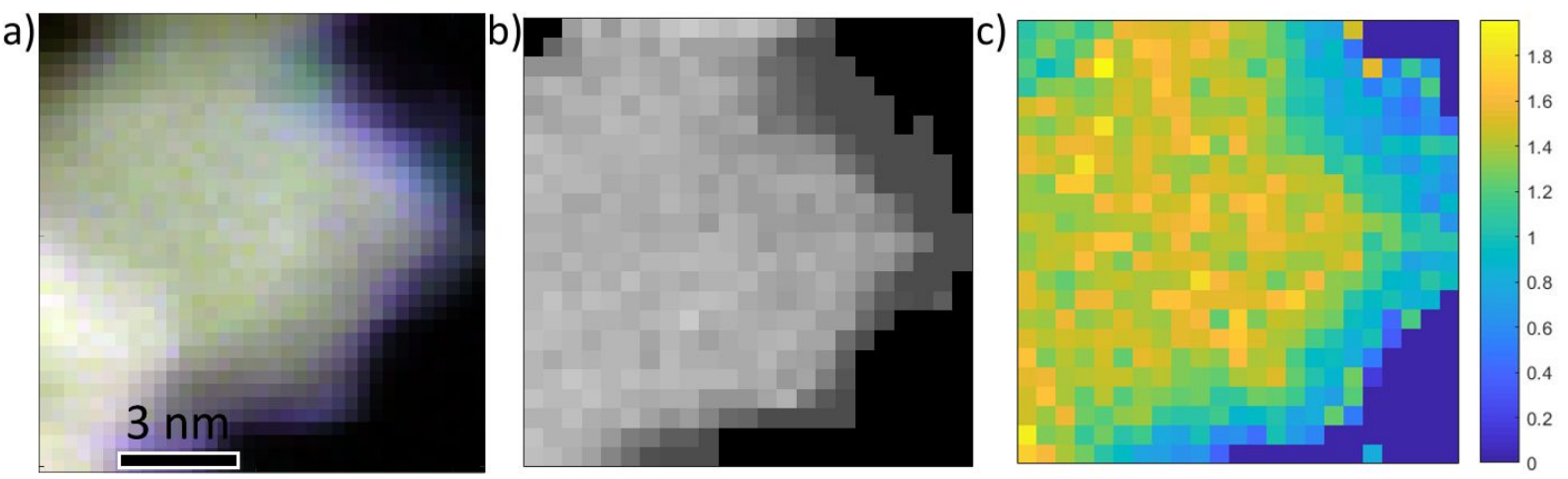

Figure S8. Chemical maps of a CS nanoparticle. a) Composition color-coded (Fe=green, Oxygen=red, Cobalt=blue) showing the Co shell. b) The shell is also visible in the Map of the Fe- $L_{3}$ peak position (scale: black=709, white=711), which shifts to lower energies with higher Co content. c) The map of the intensity ratio between O-K edge peak at $532 \mathrm{eV}$ and valley at 534 clearly reveals the Co content in the shell as well.

Geometrical phase analysis (GPA) was performed using HRSTEM images of isolated CS and CSS nanoparticles (Figure S9). A core region of the nanoparticles was used as reference area to obtain the strain maps. In the analyzed CS nanoparticles, a large central area remains almost strain free with 
strain slightly increasing towards the upper particle edges indicating a good complementarity of the crystal lattices of core and shell. On the other hand, non-negligible strains were observed throughout almost the entire CSS nanoparticle. In the lower left part of this NP it is possible to identify a crystal defect (stacking fault) that introduces strong strain $(>10 \%)$. At the edges, the very little contrast of the atomic columns results in higher noise than in the center of nanoparticles (0.5-1\%). In addition, the reference region always contains a substantial part of the shell in the direction of the projection (see Figure S4). Although a real quantitative analysis has to be taken with extreme care because of these limitations, the GPA analysis clearly reveals that strains are present.
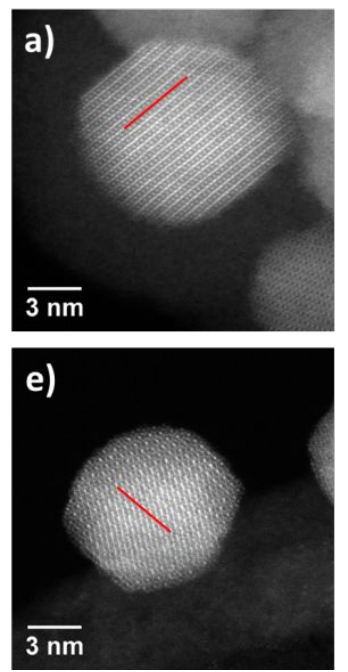
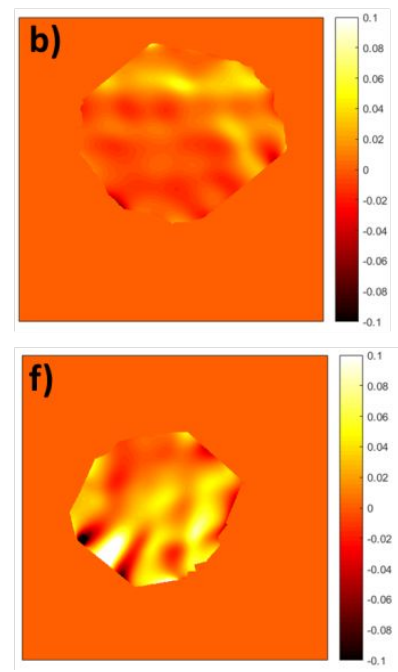
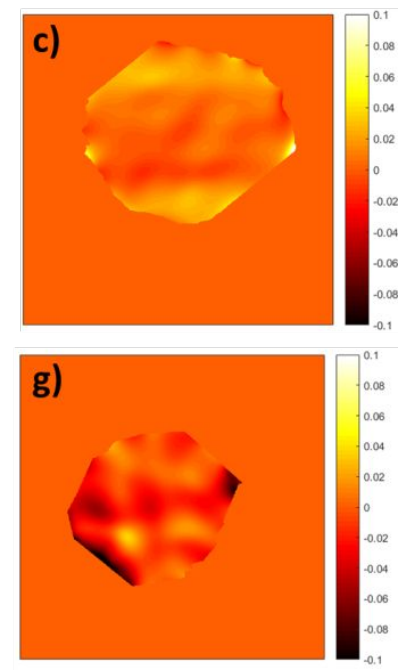
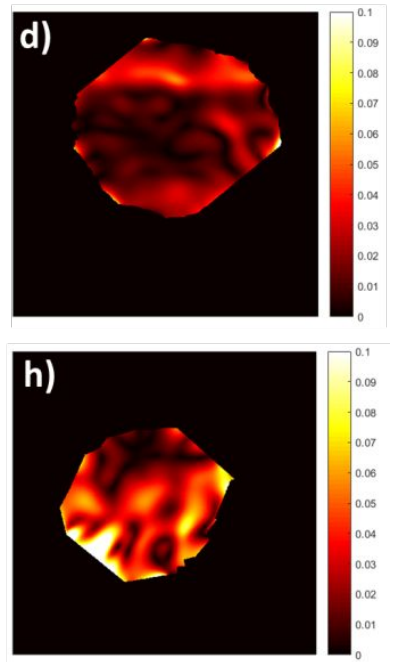

Figure 59. Geometrical Phase Analysis (GPA) of a), b), c), d) CS and e), f), g), h) CSS nanoparticles with a), e) the STEM micrographs corresponding to the strain maps b), f) parallel and c), g) perpendicular to the marked line in red. d) and $\mathrm{h}$ ) are the total amplitude. The reference region corresponds to the red line.

Fourier transform infrared (FT-IR) spectra display several bands in the range from 4000 to $450 \mathrm{~cm}^{-1}$ for C, CS and CSS (Figure S10). The bands at 2918 and $2849 \mathrm{~cm}^{-1}$ are respectively attributed to the symmetric and antisymmetric stretching vibration mode of alkyl chains $(\nu \mathrm{C}-\mathrm{H})$ from the oleic acid molecule. The bands centered at 1635 and $1414 \mathrm{~cm}^{-1}$ are attributed to the symmetric and antisymmetric stretching vibration mode of the carboxylic acid function ( $\left.v \mathrm{COO}^{-}\right)$of the oleic acid molecule grafted at the surface of the nanoparticles. ${ }^{34,91}$ The non-observation of a band at $1710 \mathrm{~cm}^{-1}$ attributed to the $\mathrm{C}=\mathrm{O}$ stretches of free carboxylic acid groups evidence the absence of remaining free oleic acid molecules, except for C. Finally, the bands at $721 \mathrm{~cm}^{-1}$ in C and CS $\left(^{*}\right)$ are attributed to the scissoring mode of the $\mathrm{H}-\mathrm{C}-\mathrm{H}$ bond of iron stearate and cobalt stearate. Although it could have been removed by further washes, we decided to not wash further the nanoparticles in order to avoid the aggregation of the nanoparticles (see granulometry measurements, Figure S11). 

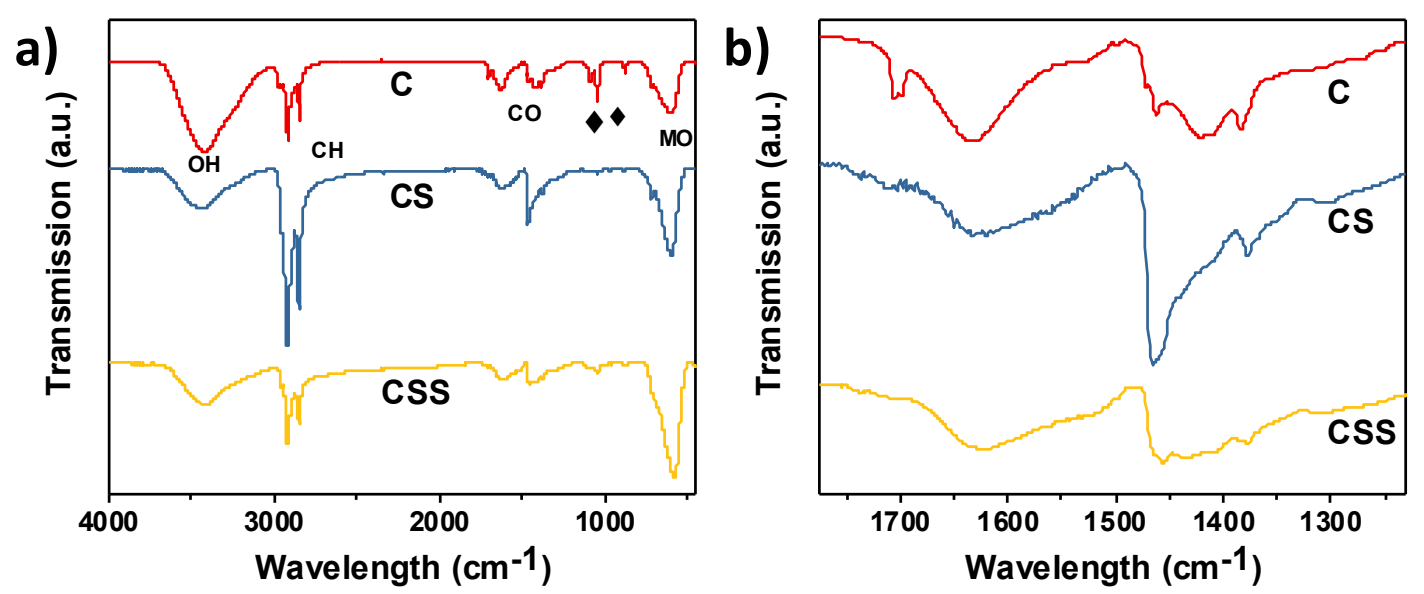

Figure S10. FTIR spectra of C, CS and CSS nanoparticles.

Thanks to the presence of oleic acid grafted at the surface of the nanoparticles, colloidal suspensions are stable in most of organic solvents such as chloroform, toluene, THF, hexane. This stability was probed by granulometry measurements performed on C, CS and CSS nanoparticles in suspension in chloroform (Figure S11). Granulometry evidenced a monomodal distribution of hydrodynamic diameters plotted as volume counts for C, CS and CSS (Figure 11a). Hydrodynamic diameters are centered to 9, 13 and $23 \mathrm{~nm}$ for C, CS and CSS, respectively. These values are larger than sizes measured from TEM micrographs because the hydrodynamic diameter includes both inorganic nanoparticles and the organic molecules grafted at their surface. Additional contributions corresponding to very small fractions were observed for CS and CSS. Intensity counts showed more clearly additional contributions to higher values, which confirmed the presence of few aggregates even for C (Figure 11b).
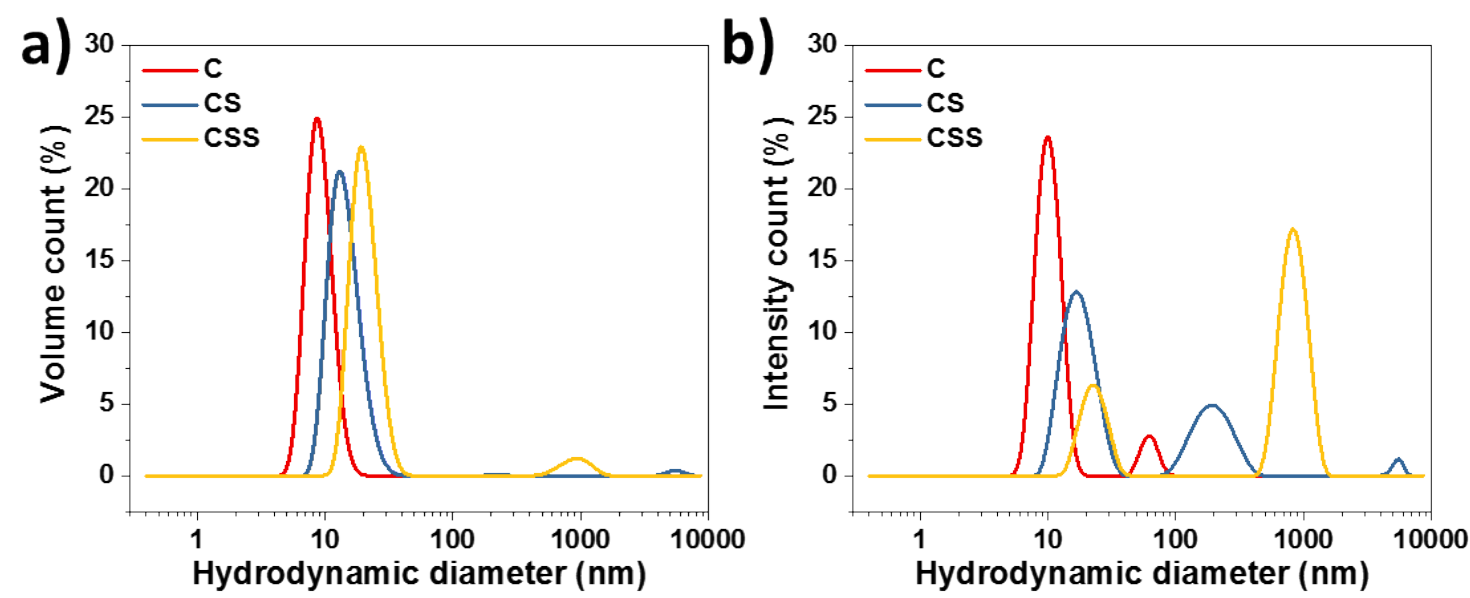

Figure S11. Distribution of hydrodynamic diameters measured for C, CS and CSS and plotted as a) volume counts and b) intensity counts. 


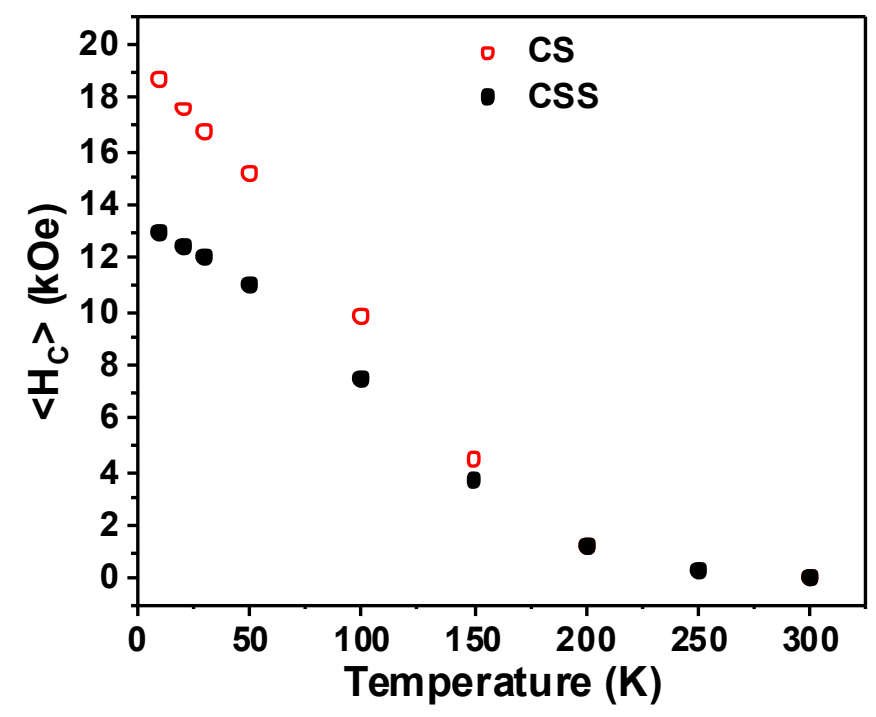

Figure S12. Temperature dependent curves of $\mathrm{H}_{\mathrm{C}}$ and for a) CS and b) CSS.

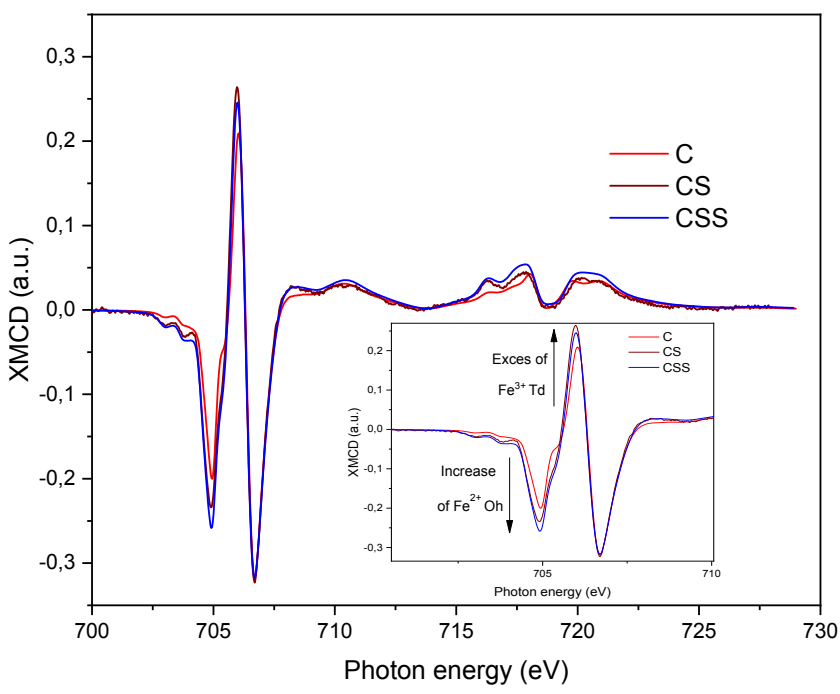

Figure S13. XMCD spectra at Fe $\mathrm{L}_{2,3}$ edges normalized at $\mathrm{S} 3$ Peak ( $\mathrm{F}^{3+}$ in $\mathrm{O}^{\text {h }}$ sites). The $\mathrm{L}_{3}$ edge region shows the gradual increase of $\mathrm{Fe}^{2+}$ in $\mathrm{O}_{\mathrm{h}}$ sites from $\mathrm{C}$, to CS and further to CSS. In addition, in the $\mathrm{L}_{2}$ region, an excess of $\mathrm{Fe}^{3+}$ in $T_{d}$ sites is observed for $\mathrm{CS}$ with respect to $\mathrm{C}$ which may result from the preference occupancy of $\mathrm{CO}^{2+}$ in $\mathrm{O}_{\mathrm{h}}$ sites. In contrast, it decreases for CSS, in agreement with the formation of the $\mathrm{Fe}_{3-\delta} \mathrm{O}_{4}$ shell. 